\title{
Effects of Universal Adhesive on Shear Bond Strength of Resin Cement to Zirconia Ceramic with Different Surface Treatments
}

\author{
Zohreh Moradi $\mathbb{D}^{1},{ }^{1}$ Farnoosh Akbari $\mathbb{D D}^{2}$ and Sara Valizadeh iD $^{3}$ \\ ${ }^{1}$ Department of Restorative Dentistry, School of Dentistry, Tehran University of Medical Sciences, Tehran, Iran \\ ${ }^{2}$ Tehran University of Medical Sciences, Tehran, Iran \\ ${ }^{3}$ Dental Research Center, Dentistry Research Institute, Restorative Dentistry Department, School of Dentistry, \\ Tehran University of Medical Sciences, Tehran, Iran \\ Correspondence should be addressed to Sara Valizadeh; valizadeh_s@sina.tums.ac.ir
}

Received 25 January 2021; Revised 17 May 2021; Accepted 5 June 2021; Published 18 June 2021

Academic Editor: Zohaib Khurshid

Copyright ( $\odot 2021$ Zohreh Moradi et al. This is an open access article distributed under the Creative Commons Attribution License, which permits unrestricted use, distribution, and reproduction in any medium, provided the original work is properly cited.

\begin{abstract}
Aim. This study aimed to assess shear bond strength (SBS) of resin cement to zirconia ceramic with different surface treatments by using Single Bond Universal. Methods. In this in vitro study, 50 zirconia discs $(2 \times 6 \mathrm{~mm})$ were divided into 5 groups of (I) sandblasting with silica-coated alumina (CoJet) + silane + Single Bond 2, (II) sandblasting with CoJet + Single Bond Universal, (III) sandblasting with alumina + Single Bond Universal, (IV) sandblasting with alumina + Z-Prime Plus, and (V) Single Bond Universal with no surface treatment. Resin cement was applied in plastic tubes $\left(3 \times 5 \mathrm{~mm}^{2}\right)$, and after 10,000 thermal cycles, the SBS was measured by a universal testing machine. The mode of failure was determined under a stereomicroscope at $\times 40$ magnification. Data were analyzed using one-way ANOVA. Results. The maximum $(6.56 \pm 4.29 \mathrm{MPa})$ and minimum $(1.94 \pm 1.96 \mathrm{MPa})$ SBS values were noted in groups III and I, respectively. Group III had the highest frequency of mixed failure (60\%). Group V had the maximum frequency of adhesive failure (100\%). Conclusion. Single Bond Universal + sandblasting with alumina or silica-coated alumina particles is an acceptable method to provide a strong SBS between resin cement and zirconia.
\end{abstract}

\section{Introduction}

Due to the increased demand for cosmetic restorations, ceramic restorations have become increasingly popular in the recent years [1]. Ceramics can be divided into two groups of silica ceramics such as feldspathic, leucite-reinforced and lithium disilicate-reinforced ceramics, and nonsilica ceramics such as alumina and zirconia [2]. Zirconia is zirconium dioxide, which was first used in orthopedics. Zirconia has gained growing popularity for dental applications in the recent years due to its favorable optical and mechanical properties and excellent biocompatibility. It can thus serve as an alternative to metal-ceramic restorations. The bond strength of zirconia depends on surface roughness and type of bonding agent used. Chipping and debonding are the most commonly reported shortcomings of zirconia restorations [3]. Debonding may occur due to the inadequate preparation of the abutment tooth, inappropriate bonding agent or cement selection, or wrong application technique $[4,5]$. Unlike silica ceramics, zirconia does not have a glass phase. Thus, it cannot be etched with hydrofluoric acid [3] and requires other surface treatments such as preparation with diamond burs, sandblasting with aluminum oxide and silica-coated alumina particles (CoJet), Nd: YAG, Er:YAG, or $\mathrm{CO}_{2}$ laser irradiation, or a combination of these methods $[6,7]$.

For the bonding to zirconia, chemical adhesives are also required in addition to surface preparation, such as silane, and phosphate-containing primers or adhesives such as 10methacryloyloxydecyl dihydrogen phosphate (10-MDP), 4methacryloxyethyl trimellitate anhydride, and thiophosphoric methacrylate. They can cause chemical reactions with zirconia oxides and enhance the bond strength as such $[8,9]$. Resin cements are the best adhesives for cementation of zirconia restorations and achieving optimal marginal adaptation, retention, and fracture resistance [10]. 
Use of different bonding agents with several application steps often confuse the clinicians, highlighting the need for simplification of the procedural steps, enhancing the bonding durability, and decreasing the technical sensitivity of the bonding procedure [11]. At present, universal adhesives are available in the market, which can bond to different types of metals and ceramics according to the manufacturers' claims. Also, they can optimally bond to dentin and enamel and can be used in self-etch, etch-andrinse, and selective-etch modes [11].

Amaral et al. [11] evaluated the shear bond strength (SBS) of Scotchbond Universal to zirconia and reported that the SBS of this universal adhesive was higher than that of Z-Prime Plus, AZ Primer, and Monobond Plus. Sharafeddin and Shoale [10] compared the SBS of All Bond Universal with the conventional bonding agents to zirconia and reported that Z-Prime Plus along with sandblasting yielded higher SBS than the universal adhesive.

Considering the controversy $[12,13]$ in the reported results regarding the SBS of universal adhesives to zirconia and the novelty of universal adhesives, further studies are warranted on this topic. Thus, the purpose of this study is to compare the SBS of Single Bond Universal to zirconia ceramic with different surface treatments in comparison with Single Bond 2 and Z-Prime Plus.

\section{Materials and Methods}

2.1. Preparation of Zirconia Specimens and Surface Treatments. In this in vitro, experimental study, 50 zirconia discs measuring $6 \times 2 \mathrm{~mm}^{2}$ were fabricated. For this purpose, presintered zirconia ceramics (CAD/CAM, Incoris Sirona, Hanau, Hesse, Germany) were sectioned by the Cercon system (Degudent, Dentsply International Company, Hanau, Hesse, Germany). All specimens were then sintered at $1550^{\circ} \mathrm{C}$ and polished with 600 -grit carbide paper. The specimens were then randomly divided into 5 groups $(n=10)$ as follows:

Group I: ten discs were sandblasted with $30 \mu \mathrm{m}$ silicacoated alumina particles (CoJet; 3M ESPE, St. Paul, $\mathrm{MN}$, USA) under 2 bar pressure at $10 \mathrm{~mm}$ distance for $15 \mathrm{~s}$ using an intraoral sandblaster (Bio Art, Barcelona, Spain). The nonsandblasted surface of specimens was marked in all specimens. Next, silane (Bisco Inc., Schaumburg, IL, USA) was applied on the surface, allowed $1 \mathrm{~min}$, and dried with air spray for $10 \mathrm{~s}$ such that its surface was no longer shiny. Then, Single Bond 2 (3M ESPE, St. Paul, MN, USA) was applied by a microbrush and after 15-20 s, it was dried with gentle air spray for $5 \mathrm{~s}$. The specimens were then light-cured (Woodpecker, Henan, China) with $450 \mathrm{~nm}$ wavelength and $1000 \mathrm{~mW} / \mathrm{cm}^{2}$ energy density (measured by a radiometer) for $10 \mathrm{~s}$.

Group II: ten discs were sandblasted with $30 \mu \mathrm{m}$ silicacoated alumina particles (CoJet; 3M ESPE, St. Paul, $\mathrm{MN}, \mathrm{USA}$ ) under 2 bar pressure at $10 \mathrm{~mm}$ distance for $15 \mathrm{~s}$ using an intraoral sandblaster (Bio Art, Barcelona, Spain). Single Bond Universal (3M ESPE, St. Paul, MN,
USA) was then applied by a microbrush and after $15-20 \mathrm{~s}$, it was dried with gentle air spray for $5 \mathrm{~s}$. Another coat of bonding agent was applied on the surface and slightly dried by $5 \mathrm{~s}$ of gentle air spray. It was then light-cured (Woodpecker, Henan, China) with $450 \mathrm{~nm}$ wavelength and $1000 \mathrm{~mW} / \mathrm{cm}^{2}$ energy density for $10 \mathrm{~s}$.

Group III: ten specimens were sandblasted with $50 \mu \mathrm{m}$ alumina particles (AL2O3, Henry west, Germany) under 2 bar pressure at $10 \mathrm{~mm}$ distance for $10 \mathrm{~s}$ using a laboratory sandblaster (Mestra, Germany). Then, Single Bond Universal was applied as explained earlier.

Group IV: ten specimens were sandblasted with $50 \mu \mathrm{m}$ alumina particles (AL2O3, Henry west, Germany) under 2 bar pressure at $10 \mathrm{~mm}$ distance for $10 \mathrm{~s}$ using a laboratory sandblaster (Mestra, Germany). Next, Z-Prime Plus (Bisco Inc., Schaumburg, IL, USA) was applied by a microbrush in two layers for $15 \mathrm{~s}$. After applying each layer, it was dried with gentle air spray for $15 \mathrm{~s}$.

Group V: Single Bond Universal was applied on the surface of the remaining 10 specimens as in groups II and III. This group served as the control group and did not receive any surface treatment.

Next, plastic tubes measuring $5 \mathrm{~mm}$ in diameter and $3 \mathrm{~mm}$ in height were placed on the surface and Dou-Link (Bisco Inc., Schaumburg, IL, USA) was applied in two 1.5$\mathrm{mm}$-thick increments into each tube such that after applying each increment, it was light-cured with $450 \mathrm{~nm}$ wavelength and $1000 \mathrm{~mW} / \mathrm{cm}^{2}$ energy density (measured by a radiometer) for $40 \mathrm{~s}$ from $1.5 \mathrm{~mm}$ distance.

2.2. Thermocycling. The groups of cemented zirconia specimens were separately wrapped in a sterile gauze, coded with a waterproof marker, and placed in a thermocycler (Vafaei Industrial TC-300, Iran). They underwent 10,000 thermal cycles between $5-55^{\circ} \mathrm{C}$ with a dwell time of $20 \mathrm{~s}$ and a transfer time of $10 \mathrm{~s}$.

2.3. SBS Testing. The mounted specimens underwent SBS testing in a universal testing machine (ZwickZ010/TN2A, Ulm, Germany) with a crosshead speed of $1 \mathrm{~mm} / \mathrm{min}$.

2.4. Mode of Failure. The mode of failure was determined under a stereomicroscope (Nikon, Tokyo, Japan) at $\times 40$ magnification as adhesive (at the bonding/ceramic or cement interface), cohesive (within the bonding), mixed (a combination of both), and cohesive substrate (within the ceramic or cement).

2.5. Statistical Analysis. Data were analyzed using SPSS version 24 via one-way ANOVA at 95\% confidence interval, assuming normal distribution of data. Considering the significant result, pairwise comparisons of SBS values and modes of failure were carried out using the Games-Howell 
and chi-square tests. $P<0.05$ was considered statistically significant.

\section{Results}

3.1. SBS Results. A number (8 out of 50) of specimens were debonded after thermocycling. Table 1 shows the number of remaining specimens after thermocycling. The SBS of debonded specimens was considered zero in statistical tests (pretest failure).

Table 2 shows the mean SBS of the five groups. The difference in SBS was significant among the five groups $(P=0.008)$. Thus, pairwise comparisons were performed by the Games-Howell test, which revealed significant differences between groups I $(1.96 \pm 1.94 \mathrm{MPa})$ and II $(4.54 \pm 2.18 \mathrm{MPa})(P=0.03)$ and $\mathrm{I}(1.96 \pm 1.94 \mathrm{MPa})$ and III $(6.56 \pm 4.29 \mathrm{MPa})(P=0.03)$. No significant differences were noted between groups I $(1.96 \pm 1.94 \mathrm{MPa})$ and IV $(3.39 \pm 3.14 \mathrm{MPa})(P=0.47)$, II $(4.54 \pm 2.18 \mathrm{MPa})$ and III $(6.56 \pm 4.29 \mathrm{MPa} \mathrm{MPa})(P=0.56)$, II $(4.54 \pm 2.18 \mathrm{MPa})$ and IV $(3.39 \pm 3.14 \mathrm{MPa})(P=0.78)$, and III $(6.56 \pm 4.29 \mathrm{MPa})$ and IV $(3.39 \pm 3.14 \mathrm{MPa})(P=0.27)$. Figure 1 shows the mean and standard deviation of SBS of the study groups.

3.2. Mode of Failure. Assessment of the mode of failure under a stereomicroscope revealed that the frequency of mixed failure was the highest $(60 \%)$ in group III (alumina + Single Bond Universal). The maximum frequency of adhesive failure (100\%) was noted in group V (Single Bond Universal) (Table 3).

Pairwise comparisons of the modes of failure by the chisquare test revealed a significant difference between groups III (alumina + Single Bond Universal) and V (Single Bond Universal) $(P=0.02)$. No other significant differences were noted $(P>0.05)$. Stereomicroscope images of adhesive and mixed failures are shown in Figures 2 and 3, respectively.

\section{Discussion}

Considering the increasing demand for all ceramic restorations due to their favorable esthetics and high strength, search for a restoration with ideal physical properties is still ongoing. Also, a strong bond between the resin cement and zirconia is a challenge in dental treatments [14].

This study assessed the SBS of Single Bond Universal to ceramic with different surface treatments in comparison with Z-Prime Plus and Single Bond 2. The results showed that sandblasting with different particles, and in other words, mechanical preparation before the application of different bonding agents, significantly increased the SBS. In the control group (Single Bond Universal alone with no mechanical surface preparation), all specimens were debonded after 10,000 thermal cycles, which indicates low SBS of specimens without surface treatment. The results of previous studies regarding the efficacy of different zirconia surface treatments have been controversial. However, the main principle is the need for mechanical surface treatment before the application of bonding agent [15]. Sandblasting with different particles is believed to be the most efficient surface treatment according to a number of researchers such as Akyil et al. and Hosseini et al. [16, 17]. Sandblasting effectively increases the surface roughness of ceramics and also increases the available ceramic surface area [17]. Thus, sandblasting was used in this study for mechanical surface treatment of zirconia. Ozcan [18] performed sandblasting with $30-50 \mu \mathrm{m}$ alumina particles under $0.5-2.5$ bar pressure from $10 \mathrm{~mm}$ distance for a maximum of $20 \mathrm{~s}$ to prevent damaging the zirconia surface. They suggested this protocol for sandblasting. The same protocol was adopted in this study. Erdem et al. [9] found no significant difference between sandblasting with alumina particles and CoJet and showed that they both increased the SBS. Their results were in line with our findings. In this study, the SBS was not significantly different in sandblasting with alumina and silica-coated alumina particles.

However, some studies have reported controversial results in this respect. Della Bona et al. [19] concluded that the tensile and shear bond strength after sandblasting with silica-coated alumina particles was higher than those after sandblasting with alumina particles. They explained that the zirconia surface coated with silica would provide a stronger bond to silane and resin [19]. Amaral et al. [11] reported that sandblasting with alumina yielded superior bond strength between resin cement and zirconia compared with sandblasting with silica-coated alumina. This result may be due to higher roughness or chemical interactions between the $\mathrm{Al}_{2} \mathrm{O}_{3}$ particles and primers, because primers containing MDP have affinity for metal oxides [11].

Amaral et al. and Ranjbar Omidi et al. used a universal adhesive and Z-Prime Plus, similar to our study, and reported that the universal adhesive along with sandblasting was an acceptable option for bonding of zirconia ceramics $[11,20]$. Their results were in agreement with our findings. Universal adhesives have adhesive resin components in addition to MDP that allows easier flow of the cement. They are copolymerized along with resin cement and create a strong bond. Also, universal adhesives contain silane, which decreases the surface tension of the substrates. Thus, the surface energy increases and enhances suitable bonding. Therefore, it increases the bond strength of nonsilica ceramics such as zirconia and alumina [21].

However, the results of Sharafeddin and Shoale [10] were in contrast to our findings. They reported that Z-Prime Plus along with sandblasting yielded a higher bond strength than All Bond Universal. They explained that Z-Prime Plus includes MDP and carboxylic monomers, which can chemically interact with the zirconia oxide layer. It seems that the synergistic effect of MDP and carboxylic monomers is the most probable reason for higher bond strength in this group.

This study also showed that 10,000 thermal cycles caused debonding of some specimens. In the control group (Single Bond Universal alone), all specimens were debonded. However, debonding occurred in a small number of specimens in the sandblasting + bonding agent or primer groups, In the CoJet + Single Bond 2 group (group I), 5 specimens were debonded, while 3 specimens were debonded in the sandblasting with alumina +Z-Prime Plus group. No debonded specimen was noted in groups where Single Bond 
TABLE 1: Number of specimens before and after thermocycling.

\begin{tabular}{lcc}
\hline Study groups & Before thermocycling & After 10,000 thermal cycles \\
\hline Sandblasting with CoJet + Single Bond 2 & 10 & 5 \\
Sandblasting with CoJet + Single Bond Universal & 10 & 10 \\
Sandblasting with alumina + Single Bond Universal & 10 & 10 \\
Sandblasting with alumina + Z-Prime Plus & 10 & 7 \\
Single Bond Universal & 10 & 0 \\
\hline
\end{tabular}

TABLE 2: Mean and SD of shear bond strength of the groups (MPa).

\begin{tabular}{lr}
\hline Groups & Mean \pm SD \\
\hline Sandblasting with CoJet + Single Bond 2 & $1.96 \pm 1.94^{\mathrm{a}}$ \\
Sandblasting with CoJet + Single Bond Universal & $4.54 \pm 2.18^{\mathrm{b}}$ \\
Sandblasting with alumina + Single Bond Universal & $6.56 \pm 4.29^{\mathrm{b}}$ \\
Sandblasting with alumina + Z-Prime Plus & $3.39 \pm 3.14^{\mathrm{ab}}$ \\
Single Bond Universal & $0.0 \pm 0.0^{\mathrm{c}}$ \\
\hline
\end{tabular}

Similar lower-case letters indicate absence of a significant difference while dissimilar letters indicate a significant difference.

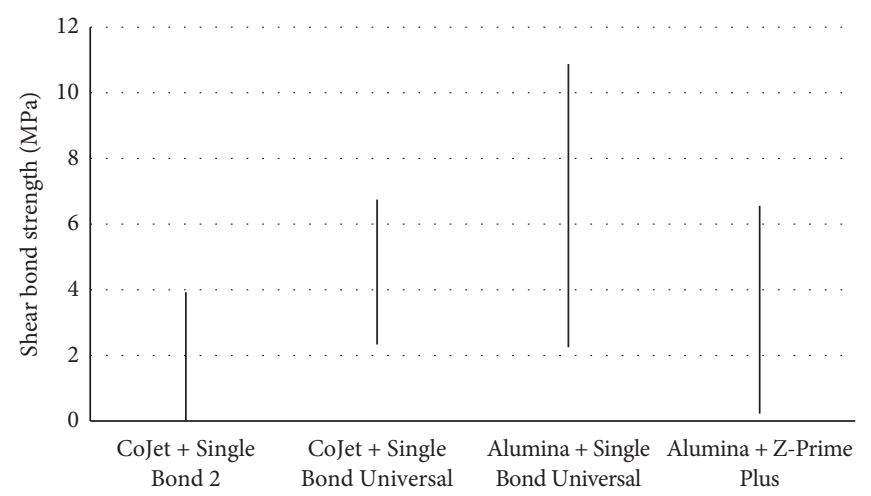

FIGURE 1: Mean and 95\% confidence interval of shear bond strength of the study groups.

TABLE 3: Frequency percentage of modes of failure in the groups.

\begin{tabular}{lccccc}
\hline & & & & Study groups \\
Sandblasting with \\
CoJet + Single Bond \\
\end{tabular}

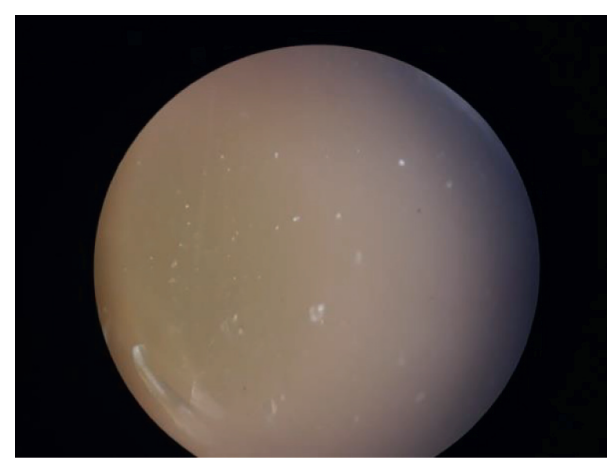

Figure 2: A specimen with adhesive failure under stereomicroscope (40x). 


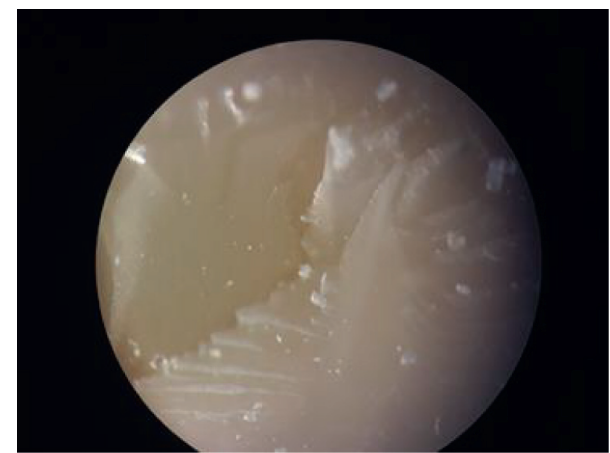

FIgURE 3: A specimen with mixed failure under stereomicroscope (40x).

Universal was used after sandblasting, which indicates high bond strength and durability following surface roughening and application of Single Bond Universal.

In the study by Amaral et al. [11], the specimens underwent 2500 thermal cycles after preparation. After thermocycling, the maximum number of debonded specimens were noted in the groups that had not received mechanical surface treatment, which was similar to our results. They also reported that universal adhesive (despite debonding of some specimens in this group) could provide acceptable bond strength even without surface treatment. In our study, the control specimens (no surface treatment and use of universal adhesive alone) were all debonded after 10,000 thermal cycles. Difference between our results and those of Amaral et al. [11] can be due to the difference in the frequency of thermal cycles, since in their study, specimens underwent 2500 thermal cycles, while this value was 10,000 cycles in our study. It seems that lower number of cycles could not significantly affect the bond strength in their study. The instruction sheet of Single Bond Universal clearly states that sandblasting is preferred for mechanical preparation before the application of bonding agent; however, a control group was also considered in our study to assess the accuracy of this statement.

The results of Ozcan, Tsuo et al., Hallman et al., and Blatz et al. were also in line with our findings. They also reported that thermocycling significantly decreased the SBS of resin cement to zirconia $[18,22-24]$. In their studies, the control group without mechanical surface treatment showed the maximum frequency of pretest failure, which indicates that increasing the micromechanical retention by sandblasting enhances the SBS. In general, according to the literature, sandblasting with alumina particles or CoJet along with the application of a bonding agent or primer-containing phosphate monomers creates a more durable bond compared with other methods. A low bond strength was noted in groups where universal bondings were used. Although sandblasting may enhance the bond strength, water sorption by Single Bond Universal, which contains a number of components, such as HEMA, 10-MDP, silane, dimethacrylate resins, filler, water, and ethanol, may decrease the bond strength after thermocycling [25].

The results of Al-Jaidi et al., Erdem et al., Chen and Shu, Atsu et al., andKumar et al. regarding the mode of failure were in line with our findings such that the majority or all failures in the control group were adhesive, whereas most failures were mixed in the sandblasted groups or when MDP-containing bonding agents were used. It seems that sandblasting before the application of bonding agent and use of primer or bonding agents containing MDP result in a strong bond between the zirconia and resin cement and consequently fewer adhesive failures [7, 9, 26-28].

\section{Conclusion}

Based on the results of this study and the limitation of this study, it was concluded that

Mechanical surface treatment is imperative before the application of universal adhesives.

The sole application of the universal bonding agent does not provide sufficient bond strength.

The high number cycles of thermocycling makes this study closer to the real clinical condition.

\section{Data Availability}

The data used to support the study are available from the corresponding author upon request.

\section{Conflicts of Interest}

The authors declare that they have no conflicts of interest.

\section{References}

[1] S. Ayyildiz, F. Emir, E. P. Tunc, and D. Sen, "Shear bond strength of various luting cements to fixed prosthodontic restorative materials," Applied Adhesion Science, vol. 3, no. 1, p. 13, 2015.

[2] R. J.-Y. Kim, J.-S. Woo, I.-B. Lee, Y.-A. Yi, J.-Y. Hwang, and D.-G. Seo, "Performance of universal adhesives on bonding to leucite-reinforced ceramic," Biomaterials Research, vol. 19, no. 1, p. 11, 2015.

[3] E. Papia, C. Larsson, M. d. Toit, and P. V. von Steyern, "Bonding between oxide ceramics and adhesive cement systems: a systematic review," Journal of Biomedical Materials Research Part B: Applied Biomaterials, vol. 102, no. 2, pp. 395-413, 2014.

[4] A. Casucci, F. Monticelli, C. Goracci et al., "Effect of surface pre-treatments on the zirconia ceramic-resin cement microtensile bond strength," Dental Materials, vol. 27, no. 10, pp. 1024-1030, 2011.

[5] P. Magne, M. P. G. Paranhos, and L. H. Burnett Jr, "New zirconia primer improves bond strength of resin-based cements," Dental Materials, vol. 26, no. 4, pp. 345-352, 2010.

[6] O. Kirmali, A. Kustarci, and A. Kapdan, "Surface roughness and morphologic changes of zirconia: effect of different surface treatment," Nigerian Journal of Clinical Practice, vol. 18, no. 1, pp. 124-129, 2015.

[7] Z. A. Al Jeaidi, M. A. Alqahtani, M. M. Awad, F. P. Rodrigues, and A. A. Alrahlah, "Bond strength of universal adhesives to air-abraded zirconia ceramics," Journal of Oral Science, vol. 59, no. 4, pp. 565-570, 2017.

[8] N. Rebholz-Zaribaf and M. Özcan, "Adhesion to zirconia as a function of primers/silane coupling agents, luting cement 
types, aging and test methods," Journal of Adhesion Science and Technology, vol. 31, no. 13, pp. 1408-1421, 2016.

[9] A. Erdem, G. Akar, A. Erdem, and T. Kose, "Effects of different surface treatments on bond strength between resin cements and zirconia ceramics," Operative Dentistry, vol. 39, no. 3, pp. E118-E127, 2014.

[10] F. Sharafeddin and S. Shoale, "Effects of universal and conventional MDP primers on the shear bond strength of zirconia ceramic and nanofilled composite resin," Journal of Dentistry (Shiraz, Iran), vol. 19, no. 1, pp. 48-56, 2018.

[11] M. Amaral, R. Belli, P. F. Cesar, L. F. Valandro, A. Petschelt, and U. Lohbauer, "The potential of novel primers and universal adhesives to bond to zirconia," Journal of Dentistry, vol. 42, no. 1, pp. 90-98, 2014.

[12] J. K. H. Tsoi, "Ceramic materials in dentistry," Advanced Dental Biomaterials, Woodhead Publishing, vol. 1, no. 1, pp. 55-78, Cambridge, UK, 2019.

[13] M. S. Zafar, R. Ullah, Z. Qamar et al., "Properties of dental biomaterials," Advanced Dental Biomaterials, Woodhead Publishing, vol. 1, no. 1, pp. 7-35, Cambridge, UK, 2019.

[14] L. R. Sofi, R. Fekrazad, M. Akbarzadeh, and M. Maleki, "Effect of Er: YAG laser, sandblast and several types of universal bonding on shear bond strength of zirconia ceramic to composite resin," The Journal of Contemporary Dental Practice, vol. 19, no. 10, pp. 1246-1253, 2018.

[15] A. L. Morresi, M. D’Amario, M. Capogreco et al., "Thermal cycling for restorative materials: does a standardized protocol exist in laboratory testing? a literature review," Journal of the Mechanical Behavior of Biomedical Materials, vol. 29, pp. 295-308, 2014.

[16] M. S. Akyil, I. H. Uzun, and F. Bayindir, "Bond strength of resin cement to yttrium-stabilized tetragonal zirconia ceramic treated with air abrasion, silica coating, and laser irradiation," Photomedicine and Laser Surgery, vol. 28, no. 6, pp. 801-808, 2010.

[17] M. H. Hosseini, F. Gorjizadeh, A. Etemadi, and P. Baghaeian, "Shear bond strength of metal brackets to zirconia treated with Er: YAG laser sandblasting and silane," Journal of Islamic Dental Association of IRAN, vol. 28, no. 3, pp. 110-117, 2016.

[18] M. Ozcan, "Air abrasion of zirconia resin-bonded fixed dental prostheses prior to adhesive cementation: why and how?" Journal of Adhesive Dentistry, vol. 15, no. 4, p. 394, 2013.

[19] D. Della Bona, M. Borba, P. Benetti, and D. Cecchetti, "Effect of surface treatments on the bond strength of a zirconiareinforced ceramic to composite resin," Brazilian Oral Research, vol. 21, no. 1, pp. 10-15, 2007.

[20] B. Ranjbar Omidi, P. K. Yeganeh, S. Oveisi, N. Farahmandpour, and F. Nouri, "Comparison of microshear bond strength of resin cement to zirconia with different surface treatments using universal adhesive and zirconia primer," Journal of Lasers in Medical Sciences, vol. 9, no. 3, pp. 200-206, 2018.

[21] S. S. Atsu, M. A. Kilicarslan, H. C. Kucukesmen, and P. S. Aka, "Effect of zirconium-oxide ceramic surface treatments on the bond strength to adhesive resin," The Journal of Prosthetic Dentistry, vol. 95, no. 6, pp. 430-436, 2006.

[22] Y. Tsuo, K. Yoshida, and M. Atsuta, "Effects of aluminablasting and adhesive primers on bonding between resin luting agent and zirconia ceramics," Dental Materials Journal, vol. 25, no. 4, pp. 669-674, 2006.

[23] L. Hallmann, P. Ulmer, F. Lehmann et al., "Effect of surface modifications on the bond strength of zirconia ceramic with resin cement resin," Dental Materials, vol. 32, no. 5, pp. 631-639, 2016.
[24] M. B. Blatz, G. Chiche, S. Holst, and A. Sadan, "Influence of surface treatment and simulated aging on bond strengths of luting agents to zirconia," Quintessence International (Berlin, Germany: 1985), vol. 38, no. 9, pp. 745-753, 2007.

[25] L. d. L. Pereira, F. Campos, A. M. D. Piva, L. D. Gondim, R. O. Souza, and M. Ozcan, "Can application of universal primers alone be a substitute for airborne-particle abrasion to improve adhesion of resin cement to zirconia?" Journal of Adhesive Dentistry, vol. 17, no. 2, pp. 169-174, 2015.

[26] L. Chen and B. I. Suh, "Bonding of resin materials to allceramics: a review," Current Research in Dentistry, vol. 3, no. 1, pp. 7-17, 2012.

[27] N. Kumar, F. Ghani, M. A. Fareed, S. Riaz, Z. Khurshid, and M. S. Zafar, "Bi-axial flexural strength of resin based dental composites-influence and reliability of the testing method configuration," Materials Technology, vol. 16, no. 2, pp. 1-7, 2021.

[28] E. P. Plueddemann, "Adhesion through silane coupling agents," The Journal of Adhesion, vol. 2, no. 3, pp. 184-201, 1970. 